\title{
Interactions between marine microorganisms and their phages
}

\author{
ZHANG Yong Yu ${ }^{1,2}$, HUANG ChunXiao ${ }^{2,3}$, YANG Jun $^{1 *} \&$ JIAO NianZhi2 $^{2 *}$ \\ ${ }^{1}$ Key Laboratory of Urban Environment and Health, Institute of Urban Environment, Chinese Academy of Sciences, Xiamen 361021, China; \\ ${ }^{2}$ State Key Laboratory of Marine Environmental Science, Xiamen University, Xiamen 361005, China; \\ ${ }^{3}$ Xiamen No. 1 High School of Fujian, Xiamen 361003, China
}

Received December 23, 2010; accepted March 30, 2011; published online May 10, 2011

\begin{abstract}
Viruses are the most abundant biological entities in marine ecosystems. Most of them are phages that infect bacteria and archaea. Phages play important roles in causing the mortality of prokaryotic cells, structuring microbial communities, mediating horizontal gene transfer between different microbes, influencing the microbial food web process, and promoting biogeochemical cycles (such as $\mathrm{C}, \mathrm{N}$, etc.) in the ocean. Here we provided an overview of recent advances in research on the interactions between marine microorganisms and their phages, and suggest future research directions based on our understanding of the literature and our own work.
\end{abstract}

marine phage, phage infection, horizontal gene transfer, phage resistance

Citation: Zhang Y Y, Huang C X, Yang J, et al. Interactions between marine microorganisms and their phages. Chinese Sci Bull, 2011, 56: 1770-1777, doi: $10.1007 / \mathrm{s} 11434-011-4503-2$

Marine virology, of which the interactions between viruses and bacteria are a very important aspect, is one of the current hotspots in marine ecology studies [1]. Viruses have the greatest abundance and highest genetic diversity in marine ecosystems, and the vast majority of them are bacteriophages, which is a key factor dominating the mortality of microorganisms. Through phage infection, horizontal gene transfer, and inducement of bacterial phage-resistant mutations, marine viroplankton play important roles in regulating microbial population sizes, community structure and diversity. In addition, viroplankton affect microbial food web processes, and are involved in marine biogeochemical cycles that include carbon and nitrogen.

\section{Discovery of marine bacteriophages and their abundance}

The bacteriophage is a viral group that infects prokaryotes (including bacteria and archaea). The group was first dis-

*Corresponding authors (email: jyang@iue.ac.cn; jiao@xmu.edu.cn) covered in 1915, although it was d'Herelle who introduced the name bacteriophage in 1917 [2]. The discovery and subsequent extensive studies on bacteriophages are of great importance for the development of the life sciences. For example, the phage-infecting-bacteria experiment proved that DNA is the genetic material of life, thus promoting the new discipline-molecular biology [2]. However, studies on marine phages began much later. In the 1990s, electron microscopic observations revealed the abundance of viral particles, mainly phages, in the sea [3]. This stimulated interest in their potential ecological roles in marine ecosystems.

The total amount of bacteriophage in the ocean is estimated to be ca. $10^{30}$. To quote Curtis A Suttle [4], a wellknown marine virologist, "if the marine viruses were connected end with end in a row, the length of this row will be longer than the distance between each of the 60 galaxies neighboring the earth". Bacteriophages exist everywhere in the ocean and the existence of microorganisms will always be accompanied by the presence of bacteriophages. In surface seawater, there are about $10^{7}$ bacteriophages per $\mathrm{mL}$, about 5-25 times than the abundance of bacteria [3]. Although viral abundance decreases with increasing water 
depth in the ocean, in sea floor sediments the number of viruses can reach a very high level of around $10^{8}-10^{9}$ per $\mathrm{cm}^{3}$. This is accompanied by a very high abundance of host bacteria because of sufficient nutrient supplies in this special environment [5]. Bacteriophages, therefore, are probably the most abundant biological organisms in the ocean.

\section{Basic characteristics and categorization of marine phages}

Marine phages have a very high genetic diversity, whereas they are not morphologically diverse. Figure 1 summarizes typical morphological types of marine phage isolated from sea water. Most of them have a compound structure with a head and tail, and contain linear double-stranded DNA (dsDNA). Using tail morphology, phages can be divided into the Siphoviridae, Podoviridae and Myoviridae [5]. The Myoviridae (Figure 1(a)) have a thick, strong and retractable tail, and always have a strong lytic ability, i.e. most of them are virulent phages. In addition, they usually have a broad host range, and can be easily isolated from seawater. Podoviridae phages (Figure 1(b)) usually have a short and non-retractable tail. They also have a relative strong lytic ability, but their host range is very small. When infecting bacteria, the Podoviridae show strict host specificity, and therefore fewer of them can be isolated from seawater. Siphoviridae phages (Figure 1(b)) usually have long tails, but unlike Myoviridae, their tails are not retractable. Moreover, their lytic ability is relatively weak. Most of them are moderate phages that cannot immediately lead to the lysis of host bacteria, but incorporate their own genome into the host genome and proliferate synchronously with the host bacteria. Their host range is between that of Myoviridae and Podoviridae. They can be isolated from seawater more
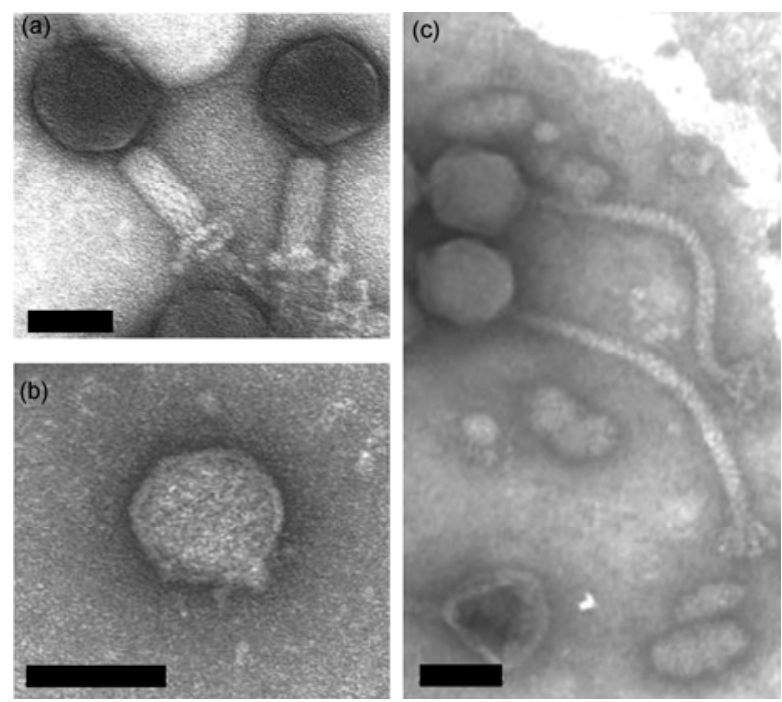

Figure 1 Three main morphological types of marine bacteriophages. (a) Myoviridae; (b) Podoviridae; (c) Siphoviridae; scale bars = $50 \mathrm{~nm}$ [5]. often [5].

\section{Functions of phages in microbial food loops and marine elemental recycling}

More and more studies have shown that bacteriophages are one of the dominant factors controlling the mortality of marine bacteria. In surface seawater, phage induced bacterial mortality can reach $10 \%-50 \%$ [6-10]. This is nearly equal to that caused by zooplankton predation. In environments not suitable for the survival of protozoa (e.g. deep-sea and sediments), phage-mediated mortality rates can reach 50\%$100 \%$. For example, a recent study performed by Danovaro and other researchers on phage lethal effects in marine sediments and the overlying water of the Atlantic, South Pacific, the Mediterranean and the Black Sea revealed that the average phage-mediated mortality rate could be $80 \%$ [11].

Since Azam put forward the concept of the "microbial food loop" in 1983, the consideration of bacteria as a central component of aquatic webs has been generally accepted. In the microbial food loop, the dissolved organic carbon (DOC) released from algae can be absorbed by heterotrophic bacteria, resulting in some photosynthetic products existing as DOC to be converted into particulate organic carbon (POC). The POC-form carbon can be consumed by small ciliates and heterotrophic flagellates and returned to the main food chain $[12,13]$. Involvement of phages makes element and energy flow in microbial food loops more complicated. It is unlike predation that transfers bacterial production and biomass to higher levels of a marine food web. Besides generating cell debris and progeny phages after being lysed by phages, bacteria will release a large amount of intracellular dissolved organic matter (DOM) to the surrounding environment. This DOM mainly includes nucleic acids and proteins, which are rich in carbon, nitrogen and phosphorus. These substances can then be reutilized by other bacteria. Bacterial production and nutrients are returned to, or maintained, at the bacteria level, and therefore forms a viral shunt in the "microbial food loop", which affects microbial food webs enhancing circulation of elements such as carbon and nitrogen among microorganisms [4]. About 1/4 of marine carbon will be circulated within the viral shunt of the microbial food loop.

In modern marine ecology, bacteria are considered as drivers for the cycles of elements and energy in marine ecosystems. Because phages can cause the death of large numbers of bacteria every day and change nutrient flow direction, they have great impacts on entire marine ecosystems [5,11]. It was reported that $6 \%-26 \%$ of the carbon fixed by photosynthesis returning to the marine DOM pool was through the effect of "viral loops", and the carbon in the marine DOM pool is equal to that in the atmosphere. Thus, it is clear that the activities of marine phages have 
important influences on global carbon cycles [4]. In addition, phages not only influence the distribution and deposition of particles in marine systems, but also regulate global climate by promoting the generation of dimethylsulfide $[3,5]$.

The lysis of microorganisms caused by phages can lead to the release of dissolved organic matter (which includes monomers, oligomers, multimers, colloidal substances and cell debris, etc.) into the surrounding environment, and prevents the deposition of dead microorganisms as particles. This in turn helps preserve some restrictive nutrients (e.g. $\mathrm{N}, \mathrm{P}$, and $\mathrm{Fe}$ ) in the euphotic layer, which is especially important for the growth of eukaryotes. Among them, Fe plays a very important role in marine ecosystems. By lysing the host cells, a large number of trace nutritive elemental complexes, such as ferric complexes, can be stored in the euphotic layer because of their dissolvability. This enhances the availability of these nutrients and maintains primary production in the euphotic layer [1].

Many marine algae have the ability of synthesizing and accumulating dimethyl-sulfoniopropionate(DMSP). By infecting the host cells, viruses can promote the release of DMSP from algae. In marine environments, DMSP can easily be degraded into the volatile sulfur compound, dimethyl sulfide(DMS), which is not only associated with the formation of acid rain and acid fog, but also can form cloud condensation nuclei increasing sunlight reflection. Therefore marine viruses also play important roles in climate regulation [12].

\section{The regulation of microbial community structure by phages}

The lethal capability of phages not only significantly affects microbial population sizes, but also changes their structure in different ways. Their mode of action mainly involves phage infection, "kill the winner", horizontal gene transfer and induction of host resistant mutations against phages [1], among which "kill the winner" has a greater impact in regulating the structure of the microbial community [14].

As different phages have distinct host specificity when infecting bacteria, their specific infection will cause the death of certain bacterial groups, thus regulating the relative abundance and proportion of different microbial populations in the marine environment. Currently, there are several hypotheses of how microbial community structure is regulated by bacteriophages, of which "kill the winner" is the most influential one. The process of phage adsorption and infection is considered a random collision process, and the frequency of successful infection is correlated with host density. When one kind of bacteria has a very low abundance in the environment, their contact rates with phages will be very low, thus few of them can be infected by phages. Those dominant bacterial species with high abundances in an environment will be more vulnerable to phage infection be- cause of their high contact rates with phages [15]. In this way, phages can regulate microbial community composition by controlling the excessive multiplication of a single specific species. Furthermore, the high phage infection frequency of dominant species will lead to an increased release of pregnant phages, which in turn, will result in more phage infection, accelerating the demise of these dominant bacterial species. The massive death of these bacterial winners will then provide necessary living space and nutrients for the growth of other bacterial groups, thus balancing the abundance of varied microbial populations and maintaining microbial diversity in a certain ecosystem. Using dilution and denaturing gradient gel electrophoresis (DGGE) methods, Zhang et al. [16] studied the influence of phage infection on microbial population abundance and structure in the coastal water of Hong Kong, China. They found that although microbial abundance decreased significantly under the pressure of phage infection, diversity increased when compared to the control without phage infection. This suggested that marine phages play important roles in maintaining the diversity of bacterial populations.

During long-term interactions between a virus and its host, a reciprocal genetic evolution mechanism is established, i.e. horizontal gene transfer [1,12]. On the one hand, when host cells are infected by phages, some mistaken cuts induced by phages will often occur in the bacterial genome, making phages carry parts of the host genes, thus the phage genome is modified. When these progeny phages carrying host genes infect other bacteria, genetic transduction will occur. On the other hand, in the lytic process, a large number of host genes will be released as free genetic fragments drifting in the water. Via transformation, they can join other host cells and change their genetic composition. During the long-term process of evolution, bacteria and phages have developed towards a more diversified direction through horizontal gene transfer, and have increased microbial community structure and functions.

During the long-term "arms race" between phages and bacteria, the host cells can modify their own gene composition to fight against phage infection [17]. However, during this process, the metabolic capacity (competitiveness) of bacteria is usually reduced to some extent because of the genetic mutation, i.e. there is a "trade-off" between the phage resistant mutation and competitiveness for host cells, thus ensuring the coexistence of mutant and wild-type strains in certain environments. This increases the structural and functional diversity of microbial populations [18]. For example, Middelboe et al. [19] mixed a marine bacterium Cellulophaga baltica MM\#3 and two phages $\left(\Phi \mathrm{S}_{\mathrm{M}}\right.$ and $\left.\Phi \mathrm{S}_{\mathrm{T}}\right)$ that can infect them in a chemostat cultivation system. After three weeks of cultivation, when the bacteria almost reached the steady state, they found that the bacteria living in the chemostat had changed to a different microbial community dominated by the phage $\Phi S T$ and $\Phi S_{\mathrm{M}}+\Phi \mathrm{S}_{\mathrm{T}}$ resistant cells, only accompanied with a small part of the previous wild 
type bacteria. Meanwhile, it was found that these mutated bacterial cells displayed significantly different metabolic activities for various carbon sources. These results revealed the important roles of marine phages in promoting the diversification of microbial communities.

\section{Phage infection mechanisms and their different survival strategies}

The interactions between marine phages and host bacteria are firstly reflected by the various phage survival strategies. Phages cannot survive independently because of their lack of a complete metabolic system. They depend on the host bacteria by using the host enzymatic systems to obtain materials and energy for proliferation [10]. To maximize the sustainable use of host bacteria, phages have evolved a variety of survival strategies.

Lytic infection is one of the most typical survival strategies. The phages relying on such a survival mechanism are called virulent phages. They can quickly complete proliferation by using the host enzymatic system, and cause the release of large amounts of progeny phages in a short time. Virulent phages are abundant in the ocean. Moebus et al. [20] reported that they accounted for $65 \%$ of the isolated viruses in the Atlantic Ocean. The lytic way is a survival strategy for phages to adapt to nutrient rich environments [2]. Under such conditions, bacterial metabolism and fission rates are accelerated, which provides basic conditions for rapid phage propagation. Furthermore this avoids extinction of host bacteria when being lysed in a large amount by phages, thus ensuring the co-proliferation of bacteria and their phages in marine ecosystems.

Lysogenic infection refers to the process of a lysogenic phage (also known as temperate bacteriophage) embedding its genome into the host chromosome, hiding in the host cell for a long time, and replicating along with the host genome. The bacteria that contain the genome of a lysogenic phage (existing as a prophage) are called lysogenic bacteria. The prophage can spontaneously, or be induced by physical or chemical factors (e.g. pH, temperature, salinity, and nutrient conditions, etc.), to deviate from the host genome, enter into a lytic cycle, resulting in the lysis of host cells and release of progeny phages. A lysogenic phage usually has more survival advantage in oligotrophic marine environments. Because nutritional restriction in these environments results in a lower abundance of host bacteria, this reduces the infection frequency by phages and prevents proliferation of virulent phages $[14,21,22]$. In a marine environment unfavorable for the survival of free phage particles, lysogenic infection will be a preferred way of surviving [23]. It was reported that the abundance of lysogenic bacteria in the open sea is much greater than that in coastal (or near-shore) water [24-27]. The reason is because of the reduced impact of human activities in the open ocean, making water much clearer than the coastal area, thus increasing the UV penetration depth, and resulting in massive death of free phage particles. For better survival, these living phage particles adopt a lysogenic way and temporarily "parasitize" the host cells to avoid the threat from adverse environments [28]. Weinbauer et al. [29] pointed out that the heterotrophic microbial community of deep seawater is mainly composed of lysogenic bacteria.

The life form of pseudo-lysogeny is widely distributed in marine ecosystems, characterized by the independent existence of phage nucleic acid in the host cytoplasm like plasmids. The detailed mechanism of this survival strategy is still controversial. Ripp and Miller [30,31] have pointed out that lysogeny is an unstable period for phages to coexist with their host cells living in starvation. During this period, phages cannot get enough energy to start gene expression for entering the lysogenic or lytic cycles. Moebus [32] considered that pseudo-lysogeny is a temporary immune state of host bacteria. The bacteria and phage can coexist during this period, unless being induced to lytic processes by the inducing factor that may be a polysaccharide depolymerase. In addition, it has been suggested that this kind of survival strategy can help marine phages take appropriate measures to face environmental changes. It provides a possible explanation of how marine phages survive in an unfavorable environment [14].

Chronic infection is characterized by phages not causing lysis of the host cells, and progeny phages being released through secretion or budding [10]. The ecological meaning of this survival strategy and under what conditions the phages will choose this special way to survive are still not very clear.

Marine phages usually maximize their proliferation by controlling the latent time in the host cells. Host abundance and their physiological status are two factors that indirectly determine the success of this process [33,34]. Prolonging the latent period will lead to more proliferation of progeny phages in the host cell. When the surrounding environment is suitable for rapid growth of the host cells, the progeny phages will be released and immediately start a new phage infection cycle in other bacteria [10]. In an environment that contains abundant host cells with high metabolic activities, phages will mainly choose the lytic way for rapid release of progeny phages by shortening the latent period [29]. By contrast, phages usually prefer the lysogenic way to extend their latent period where only few host bacteria exist in an environment, with the purpose of maximizing the release amount of progeny phages (i.e. burst size) and living through the adverse conditions [33]. Currently, burst size is a very effective parameter for evaluating the survival of phages.

With regard to the ecological strategies, most marine phages employ an r-selected strategy, which is characterized by rapid rate of proliferation, high burst size, and short generation cycle, etc. They are generally lytic phages that can 
rapidly cause the death of host cells in several minutes or hours. Another survival strategy is the K-selected way. $\mathrm{K}$-selected phages are usually lysogenic or pseudo-lysogenic phages that have small genome sizes and can integrate their DNA into host genomes or establish other carrier relationships with the host cells. Their burst sizes are usually smaller than that of r-selected phages. The third one is a strategy along the $\mathrm{r}-\mathrm{K}$ selection continuum, such as the marine phages that specifically infect SAR11 bacterial groups. In general, K-selected marine phages tend to infect the most abundant, slow-growing microbial communities. R-selected phages are usually highly virulent and tend to kill the microbial populations that are growing rapidly in response to transient, favorable conditions. Compared with other species in the sea, the high growth rate and rapid responses to environments by marine phages determine that they are more prone to the r-selection end of the r-K-selection spectrum [4].

\section{Horizontal gene transfer-co-evolutionary mechanism of microorganisms and their phages}

Marine phages are important agents for gene transfer between different bacteria [35]. When phages are assembled in donor bacterial cells, parts of the host genes (including some random DNA fragments or genes nearby the prophage integration sites) are often mistakenly packaged into the capsids. When a new infection cycle of the progeny phages is started, these mistakenly packaged genes from the previous donor bacteria will be transferred to other homogeneous or heterologous bacteria as the new gene's recipients, thus a horizontal gene transfer will take place. During this process, new genes are likely formed by integration of the extraneous genes with the original genome. This confers the possibility for bacteria to adapt to different marine environments and face various natural selection pressures [36,37]. Some prophages can even change the host phenotypes once their gene expression is started within host cells during the lysogenic lifecycle [35]. In addition, during the process of horizontal gene transfer, the phage capsid will act as a gene pool holding exogenous genetic material and protecting their activities from the enzymatic degradation by nucleases in the surrounding environment [38,39].

Because direct quantification of the frequency of horizontal gene transfer mediated by phages is not easy, model construction and molecular biology methods are employed to predict the approximate value. Methods include adding known genes into in situ marine environments to detect the successful integration frequency within natural microbial communities, or to evaluate the gene transfer rate by addition of recipient bacteria, or through genomic analysis of the isolated bacterium from marine environments. By using mathematical models, Jiang and Paul calculated that the average frequency of horizontal gene transfer at the U.S
Tampa Bay Estuary could reach as high as $1.3 \times 10^{14}$ times every year [40].

Horizontal gene transfer mediated by marine phages is influenced by many factors. First, when the recipient bacteria are in the lysogenic state while the donor bacteria are not, horizontal gene transfer will occur more frequently. Second, it is related to the host specificity of phages. The phages with a wider host range are more important for horizontal gene transfer. Moreover, temperature can influence the occurrence of horizontal gene transfer. For example, horizontal gene transfer will not take place between mesophilic and thermophilic bacteria. This is because under high temperature mesophilic proteins will lose their activity, whereas under low temperature the enzymatic activities of thermophilic bacteria will be inhibited [41]. In addition, some other factors such as MOI (multiplicity of infection), bacterial genome size, and G/C content, etc., would also affect the occurrence of horizontal gene transfer [40,41].

Marine phages can lead to the release of DNA, cell wall, cytoplasm and other structural materials during host lysis. The released bacterial DNA enters into the environment, and will be partly degraded by nucleases, or be used by other phages to synthesize their own DNA [10]. However, there is still a lack of more in-depth studies on quantification of the released DNA, as well as the phage's effects on energy flow within the marine microbial food loop.

The genome of marine bacteria is highly dynamic and mosaic. Lots of genetic information can be constantly inserted or removed from the genome by horizontal gene transfer [10]. Via phages, the genetic material from donor bacteria can be widely spread among the recipient bacterial populations. Under natural selection, only those genes which are beneficial to the recipient bacteria can be preserved [42]. This process will have important effects on both the donor and recipient bacteria, finally resulting in the generation of novel bacterial species and the increase of genetic diversity of microbial communities [36,43]. Meanwhile, the genotypes of marine phages will also constantly evolve towards diversification because of the acquirement of host genes. It is estimated that there are as many as 5000 different kinds of phages in each $100 \mathrm{~L}$ of sea water [44]. During long-term interactions between marine bacteria and their phages, they mutually benefit each other and realize their coevolution. Lindell et al. [45-47] found that the phages infecting the marine cyanobacteria Synechococcus and Prochlorococcus usually carry a gene which can encode the photosynthetic protein (psbA). When infecting the host bacteria, the phage psbA gene and other related photosynthetic genes could be expressed inside the host cells to keep cell activity, and in reverse to provide necessary energy for the proliferation of phages themselves. Besides, bacteria can also accept some phage genes useful to themselves during the long-term evolution to enhance their own competitiveness. For example, there are two phage tail gene clusters in the bacterium Pseudomonas aeruginosa, which can encode 
bacteriocin inhibiting the growth of other bacteria, thus improving their own competitiveness in certain environments [48].

\section{Phage resistance mechanisms of marine bacteria}

At an infection pressure of up to $10^{23}$ /s [4], to avoid massive death by phage infection, host bacteria have evolved a series of effective anti-phage mechanisms. At present, there are five anti-phage mechanisms that have been reported, including adsorption inhibition, DNA penetration blocking, restriction/modification, abortive infection, and clustered regularly interspaced short palindromic repeats (CRISPRs) $[10,49,50]$. Some of these mechanisms exercise their functions respectively at different key steps during the phage generation cycle, including adsorption, injection, biosynthesis, assembling, and release, to block the generation of progeny phages (Figure 2). These defensive mechanisms against phages are also effective for bacteria to control the abundance of phage populations [51].

Adsorption inhibition is the first line to defend phage infection. Without correctively recognizing the adsorption receptors, phages cannot attach well to host bacteria. Most of the receptors are teichoic acid, lipopolysaccharide molecules or glycoprotein compounds, which are located on the cell wall, pilus, flagella or capsule. By regulating the expression of receptor genes to change the steric configuration of receptors or modify receptors, host bacteria can interfere with phages to recognize their specific adsorption locus, and prevent their successful adsorption [10]. Recently, we studied the anti-phage infection mechanisms of a marine bacterium Roseobacter denitrificans OCh114 which has an aerobic anoxygenic phototrophic ability, and found that the ex- pression of some bacterial surface membrane proteins were significantly down-regulated. We hypothesized that the down-regulated membrane proteins may serve as potential phage receptors whose absence prevented the adsorption of phages to host cells and subsequent infection [52].

Phage DNA injection blocking refers to that after the phage adsorbs well on the host bacterium. The bacterium prevents the phage injecting its DNA into the host cytoplasm by changing the surface permeability. So far, the detailed mechanisms about DNA injection blocking are still not very clearly understood $[10,17,53]$.

When phage DNA is injected successfully into the cytoplasm, the host bacterium may start the restriction/modification defense system to prevent the phage DNA from integrating with its own chromosome. The restriction/modification system is composed mainly of restriction endonuclease and methylase. It can cut the extraneous DNA into fragments, but does not affect its own DNA [54]. When phage DNA enters the host cell, the restriction/modification system will recognize the extraneous DNA sequence and cut them into fragments at the recognition locus. Later, these DNA fragments can be further degraded by other endonucleases. However, because host DNA has been methylated in advance by the bacterial methylase, it can escape recognition by its own restriction endonuclease. However, there are also a few phages whose DNA cannot be recognized by the host restriction endonuclease. This part of extraneous phage DNA will be mistakenly considered by host cells as their own DNA and will be then protected via methylation. Therefore the restriction/modification system is considered as the most powerful, but also the most vulnerable, defense system [54]. Moreover, some phages evolved specific strategies to defend against the destruction by host restriction endonuclease, such as removing the restriction loci within phage DNA, obtaining their own meth-

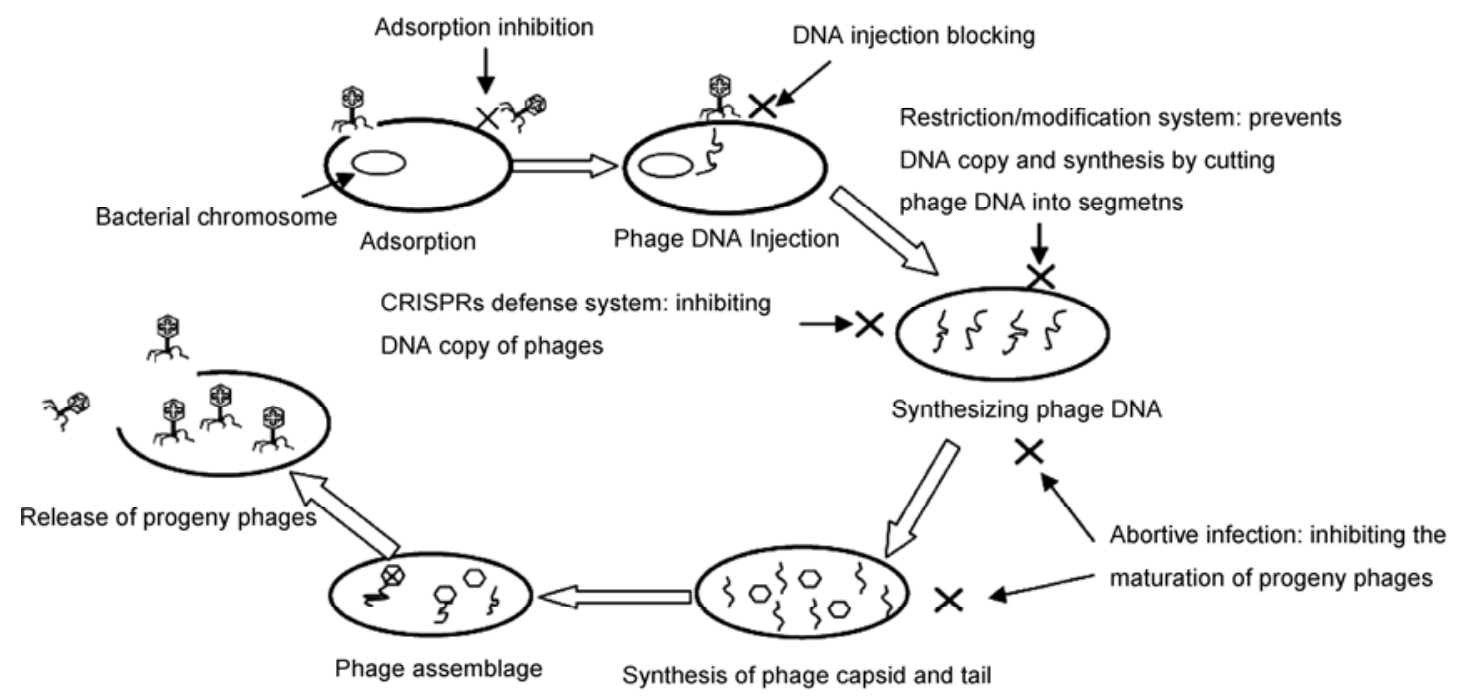

Figure 2 The complete phage infection process and the host anti-phage defense strategies at different stages during the phage infection cycle. 
ylation gene, modifying the receptor molecules by methylation or glycosylation, or synthesizing proteins that can resist the host's restriction endonuclease $[10,14]$.

Abortive infection is a special way for those bacteria having "suicidal sacrifice" spirits to defend against phages. It suppresses phage development by interfering with its DNA replication and structural assemblage [10]. This suicidal-sacrifice phage resistance can prevent bacteria of the same population from infection by phages. Therefore, the development of suicide genes in bacteria seems rational from an evolutionary perspective $[31,54]$.

The defense system of CRISPRs is a novel prokaryotic phage resistance mechanism discovered recently. It is widely distributed in bacteria and achaea. CRISPRs contain multiple short direct repeats. Short segments of foreign DNA, call spacers, are incorporated into the genome between CRISPR repeats [49,50]. When these exogenous DNA are homologous to the DNA sequences of some kind of phages, the host cell will embody its resistance against these phages [55-57]. CRISPRs function as a prokaryotic immune system in a manner analogous to RNAi in eukaryotic organisms [49,58].

Besides the five typical anti-infection mechanisms mentioned above, there are some new findings reported recently regarding the anti-phage resistance mechanisms. For example, proteomics was firstly employed to analyze the variations in protein expression of a marine roseobacter Silicibacter pomeroyi DSS-3 after acquiring the anti-phage ability, and found that the alkaline modification of four intracellular proteins may contribute to the phage resistance of host cells [59]. Although these proteins and their detailed modification type were not successfully characterized, it indicated that alkaline modification of some key intracellular proteins is likely an unknown strategy for the host cell to defend against phages. We suggest that along with the technological development for protein and modification characterization, more and more novel phage resistance mechanisms will be discovered in the future.

\section{Prospects}

With the development of marine virology, researchers have recognized that the interactions between phages and bacteria are complex processes in marine ecosystems, and that marine phages play important roles in regulating microbial communities, promoting microbial evolution, and accelerating microbial food loop dynamics. However, the detailed mechanisms of the dynamic interactions between bacteria and phages are not very clear. We consider that the following aspects are still worth more in-depth exploration in the future. (1) Combining field studies with mesocosm experiments to carry out larger scale ecological surveys that include estuaries, near and off shore of different sea areas, coastal water, open ocean, and also at different depth ranges within them. In addition, the studies should examine the ecological dynamics (including abundance, community composition, biological activities, and so on) of bacteria and phages under different environmental conditions (such as different temperature, salinity, light and water pressure). This should help for a complete understanding of the important roles of marine phages in controlling microbial growth rates or mortality, and the regulation of microbial community structure and diversity. (2) Reevaluating phage impacts on energy flow within the microbial food loop through accurately quantifying the released structural materials from bacteria. (3) Studying in situ phage communities in the marine environment by using a combination of environmental genomics and proteomics, for the purpose of understanding the structural and functional diversity of marine phage communities. (4) At the gene and protein level, further studying the coevolution of marine phages and bacteria by using advanced molecular biology techniques, such as genomic and proteomics analysis.

This work was supported by the National Natural Science Foundation of China (41006087, 91028001 and 41076063), the MEL Young Scientist Visiting Fellowship (MELRS0931) and the Key Science and Technology Project of Fujian Province, China (2009Y0044).

1 Jiao N Z. Marine Microbial Ecology (in Chinese). Beijing: Science Press, 2006. 272-303

2 Duckworth D H. History and basic properties of bacterial viruses. In: Goyal S M, Gerba C P, Bitton G, eds. Phage Ecology. New York: John Wiley \& Sons, 1987. 1-44

3 Fuhrman J A. Marine viruses and their biogeochemical and ecological effects. Nature, 1999, 399: 541-548

4 Suttle C A. Marine viruses-Major players in the global ecosystem. Nat Rev Microbiol, 2007, 5: 801-812

5 Suttle C A. Viruses in the sea. Nature, 2005, 437: 356-361

6 Fuhrman J A, Suttle C A. Viruses in marine planktonic systems. Oceanography, 1993, 6: 51-63

7 Steward G F, Smith D C, Azam F. Abundance and production of bacteria and viruses in the Bering and Chukchi Seas. Mar Ecol-Prog Ser, 1996, 131: 287-300

8 Weinbauer M G, Hofle M G. Significance of viral lysis and flagellate grazing as factors controlling bacterioplankton production in a eutrophic lake. Appl Environ Microbiol, 1998, 64: 431-438

9 Suttle C A. The significance of viruses to mortality in aquatic microbial communities. Microb Ecol, 1994, 28: 237-243

10 Weinbauer M G. Ecology of prokaryotic viruses. FEMS Microbiol Rev, 2004, 28: 127-181

11 Danovaro R, Dell A, Corinaldesi C, et al. Major viral impact on the functioning of benthic deep-sea ecosystems. Nature, 2008, 454: 1084 $-1087$

12 Wang F, Zheng T L, Hong H S. The important roles of marine virus in microbial food loop (in Chinese). Mar Sci, 1998, 4: 41-43

13 Wilhelm S W, Suttle C A. Viruses and Nutrient Cycles in the Sea. Bioscience, 1999, 49: 781-788

14 Wommack K E, Colwell R R. Virioplankton: Viruses in aquatic ecosystems. Microbiol Mol Biol Rev, 2000, 64: 69-114

15 Thingstad T F, Lignell R. Theoretical models for the control of bacterial growth rate, abundance, diversity and carbon demand. Aquat Microb Ecol, 1997, 13: 19-27

16 Zhang R, Weinbauer M G, Qian P Y. Viruses and flagellates sustain apparent richness and reduce biomass accumulation of bacterioplankton in coastal marine waters. Environ Microbiol, 2007, 9: 
3008-3018

17 Allison G E, Klaenhammer T R. Phage resistance mechanisms in lactic acid bacteria. Inter Dairy J, 1998, 8: 207-226

18 Bohannan B J M, Kerr B, Jessup C M, et al. Trade-offs and coexistence in microbial microcosms. Antonie van Leeuwenhoek, 2002, 81: $107-115$

19 Middelboe M, Holmfeldt K, Riemann L, et al. Bacteriophages drive strain diversification in a marine Flavobacterium: Implications for phage resistance and physiological properties. Environ Microbiol, 2009, 11: 1971-1982

20 Moebus K, Nattkemper H. Bacteriophage sensitivity patterns among bacteria isolated from marine waters. Helgoland Mar Res, 1981, 34: 375-385

21 Echols H. Developmental pathways for the temperate phage: Lysis vs lysogeny. Ann Rev Genet, 1976, 6: 157-190

22 Hewson I, O'Neil J M, Fuhrman J A, et al. Virus-like particle distribution and abundance in sediments and overlying waters along eutrophication gradients in two subtropical estuaries. Limnol Oceanogr, 2001, 46: 1734-1746

23 Lenski R E. Dynamics of interactions between bacteria and virulent bacteriophage. Adv Microb Ecol, 1988, 10: 1-44

24 Weinbauer M G, Suttle C A. Lysogeny and prophage induction in coastal and offshore bacterial communities. Aquat Microb Ecol, 1999, 18: $217-225$

25 Jiang S C, Paul J H. Occurrence of lysogenic bacteria in marine microbial communities as determined by prophage induction. Mar Ecol Prog Ser, 1996, 142: 27-38

26 Jiang S C, Paul J H. Seasonal and diel abundance of viruses and occurrence of lysogeny/bacteriocinogeny in the marine environment. Mar Ecol Prog Ser, 1994, 104: 163-172

27 Jiang S C, Paul J H. Significance of lysogeny in the marine environments: studies with isolates and a model of lysogenic phage production. Microb Ecol, 1998, 35: 235-243

28 Wilhelm S W, Weinbauer M G, Suttle C A, et al. The role of sunlight in the removal and repair of viruses in the sea. Limnol Oceanogr, 1998, 43: 586-592

29 Weinbauer M G, Brettar I, Höfle M. Lysogeny and virus-induced mortality of bacterioplankton in surface, deep, and anoxic waters. Limnol Oceanogr, 2003, 48: 1457-1465

30 Ripp S, Miller R V. The role of pseudolysogeny in bacteriophagehost interactions in a natural freshwater environment. Microbiology, 1997, 143: 2065-2070

31 Ripp S, Miller R. Dynamics of the pseudolysogenic response in slowly growing cells of Pseudomonas aeruginosa. Microbiology, 1998, 144: 2225-2232

32 Moebus K. Marine bacteriophage reproduction under nutrient-limited growth of host bacteria. 2. Investigations with phage-host system [H3:H3/1]. Mar Ecol Prog Ser, 1996, 144: 13-22

33 Abedon S T. Selection for bacteriophage latent period length by bacterial density: A theoretical examination. Microb Ecol, 1989, 18: 79-88

34 Abedon S T, Herschler T D, Stopar D. Bacteriophage latent-period evolution as a response to resource availability. Appl Environ Microbiol, 2001, 67: 4233-4241

35 Rohwer F, Prangishvili D, Lindell D. Roles of viruses in the environment. Environ Microbiol, 2009, 11: 2771-2774

36 Davison J. Genetic exchange between bacteria in the environment. Plasmid, 1999, 42: 73-91

37 Dutta C, Pan A. Horizontal gene transfer and bacterial diversity. J Bios, 2002, 27: 27-33
38 Stotzky G. Gene transfer among bacteria in soil. In: Gene Transfer in the Environment. New York: McGraw-Hill, 1989. 165-222

39 Schicklmaier P, Schmieger H. Frequency of generalized transducing phages in natural isolates of the Salmonella typhimurium complex. Appl Environ Microbiol, 1995, 61: 1637-1640

40 Jiang S C, Paul J H. Gene transfer by transduction in the marine environment. Appl Environ Microbiol, 1998, 64: 2780-2787

41 Jain R, Rivera M C, Moore J E, et al. Horizontal Gene Transfer Accelerates Genome Innovation and Evolution. Mol Biol Evol, 2003, 20: 1598-1602

42 Lawrence J G. Gene transfer, speciation, and the evolution of bacterial genomes. Curr Opin Microbiol, 1999, 2: 519-523

43 Gogarten J P, Townsend J P. Horizontal gene transfer, genome innovation and evolution. Nat Rev Microbiol, 2005, 3: 679-687

44 Rohwer F, Thurber R V. Viruses manipulate the marine environment. Nature, 2009, 459: 207-212

45 Lindell D, Jaffe J D, Coleman M L, et al. Genome-wide expression dynamics of a marine virus and host reveal features of co-evolution. Nature, 2007, 449: 83-86

46 Lindell D, Sullivan M B, Johnson Z I, et al. Transfer of photosynthesis genes to and from Prochlorococcus viruses. Proc Nat Acad Sci USA, 2000, 101: 11013-11018

47 Lindell D, Jaffe J D, Johnson Z I, et al. Photosynthesis genes in marine viruses yield proteins during host infection. Nature, 2005, 438: 86-89

48 Nakayama K, Takashima K, Ishihara H, et al. The R-type pyocin of Pseudomonas aeruginosa is related to $\mathrm{P} 2$ phage, and the F-type is related to lambda phage. Mol Microbiol, 2000, 38: 213-231

49 Barrangou R, Fremaux C, Deveau H, et al. CRISPR provides acquired resistance against viruses in prokaryotes. Science, 2007, 315: 1709-1712

50 Cui Y J, Li Y J, Yan Y F, et al. Clustered regularly interspaced short palindromic repeats: Structure, function and application (in Chinese). Acta Microbiol Sin, 2008, 48: 1549-1555

51 Chibani-Chennoufi S, Bruttin A, Dillmann M L, et al. Phage-host interaction: An ecological perspective. Bacteriol, 2004, 186: 36773686

52 Huang C X, Zhang Y Y, Jiao N Z. Phage Resistance of a Marine Bacterium, Roseobacter denitrificans OCh114, as Revealed by Comparative Proteomics. Curr Microbiol, 2010, 61: 141-147

53 Hill C. Bacteriophage and bacteriophage resistance in lactic acid bacteria. FEMS Microbiol Rev, 1993, 12: 87-108

54 Forde A, Fitzgerald G F. Bacteriophage defence systems in lactic acid bacteria. Antonie van Leeuwenhoek, 1999, 76: 89-113

55 Bolotin A, Quinquis B, Sorokin A, et al. Clustered regularly interspaced short palindrome repeats (CRISPRs) have spacers of extrachromosomal origin. Microbiol, 2005, 151: 2551-2561

56 Mojica F J M, Díez-Villaseñor C, García-Martínez J, et al. Intervening sequences of regularly spaced prokaryotic repeats derive from foreign genetic elements. J Mol Evol, 2005, 60: 174-182

57 Pourcel C, Salvignol G, Vergnaud G. CRISPR elements in Yersinia pestis acquire new repeats by preferential uptake of bacteriophage DNA, and provide additional tools for evolutionary studies. Microbiol, 2005, 151: 653-663

58 Deveau H, Barrangou R, Garneau J E, et al. Phage response to CRISPR-encoded resistance in Streptococcus thermophilus. J Bacteriol, 2008, 190: 1390-1400

59 Zhang Y Y, Jiao N Z, Colquhoun D R, et al. Protein modifications related to phage resistance in a marine roseobacter. Aquat Microb Ecol, 2009, 55: 203-207

Open Access This article is distributed under the terms of the Creative Commons Attribution License which permits any use, distribution, and reproduction in any medium, provided the original author(s) and source are credited. 